03,09, 12

\title{
Фотолюминесценция гетероструктур с ультратонкими квантовыми ямами CdTe/ZnTe
}

\author{
() Н.Г. Философоов ${ }^{1}$, А.Ю. Серов ${ }^{1}$, G. Karczewski ${ }^{2}$, В.Ф. Агекян ${ }^{1}$, Н. Mariette $^{3}$, В.П. Кочерешко \\ ${ }^{1}$ Санкт-Петербургский государственный университет, \\ Санкт-Петербург, Россия \\ ${ }^{2}$ Institute of Physics, Polish Academy of Sciences, \\ Warsaw, Poland \\ ${ }^{3}$ Institut Neel CNRS, \\ Grenoble, France \\ ${ }^{4}$ Физико-технический институт им. А.Ф. Иофффе РАН, \\ Санкт-Петербург, Россия \\ ฯ E-mail: Vladimir.Kochereshko@mail.ioffe.ru
}

Поступила в Редакцию 26 марта 2020 г.

В окончательной редакции 26 марта 2020 г.

Принята к публикации 2 апреля 2020 г.

Исследованы гетероструктуры CdTe/ZnTe с ультратонкими квантовыми ямами. В спектрах наблюдались линии легкого и тяжелого экситонов, привязанных к слоям СdTе. Обнаружено, что интенсивность фотолюминесценции легкого экситона сравнима с интенсивностью люминесценции тяжелого экситона. Температурные сдвиги этих линий не совпадали, и линии пересекались при температуре $65 \mathrm{~K}$. Проведенные оценки энергии и волновых функций экситонных состояний позволил уточнить некоторые зонные параметры и параметры экситонов в таких структурах.

Ключевые слова: квантовые ямы, экситоны, фотолюминесценция.

DOI: $10.21883 /$ FTT.2020.09.49771.30H

\section{1. Введение}

Полупроводниковые соединения $\mathrm{A}_{2} \mathrm{~B}_{6}$, в частности $\mathrm{CdTe}$ и ZnTe, часто используются для фундаментальных исследований как модельный объект. Их использование для приборных приложений незначительно из-за быстрой деградации. При этом они часто обладают высоким структурным совершенством и уникальными оптическими свойствами. Было бы заманчиво найти возможности для их более широкого практического использования.

Одна из причин, сдерживающих практическое применение гетероструктур на основе этих соединений, заключается в заметном рассогласовании кристаллических решеток. В результате на интерфейсах возникают механические напряжения, приводящие в итоге к деградации структуры. Этого недостатка лишены гетероструктуры с ультратонкими квантовыми ямами, в которых решетки „Подстраиваются“ друг к другу [1]. Однако из-за рассогласования решеток величина разрыва зон известна лишь приближенно. Так, разброс опубликованных данных по величине разрыва зон в валентной зоне гетероструктур $\mathrm{CdTe} / \mathrm{ZnTe}$ достигает $\pm 10 \%$ от общего разрыва зон [2]. Еще хуже обстоит дело со структурами, содержащими ультратонкие (до моноатомных) слои. Для таких структур само понятие разрыва зон становится расплывчатым [3].

Разрыв зон складывается из химического разрыва зон, который определяется химическим строением интерфейса, и деформационного разрыва зон, который связан c упругой энергией на интерфейсе контактирующих материалов. В соответствии с правилом „общего аниона/катиона“ в структурах с общим анионом разрыв зон в валентной зоне должен быть мал. Деформация может дополнительно уменьшить разрыв зон и даже привести к образованию структуры типа-II для дырок. В этом случае главным для квантования движения дырки становится ее кулоновское взаимодействие с электроном.

В настоящей работе проведено детальное экспериментальное исследование спектров фотолюминесценции структур CdTe/ZnTe с ультратонкими квантовыми ямами. В результате удалось уточнить величины разрыва в валентной зоне для таких структур и уточнить некоторые параметры экситонов.

\section{2. Эксперимент}

В работе исследовались образцы, представляющие собой слои ZnTe толщиной в несколько десятков микрон. Примерно в центре образца вместо двух монослоев атомов $\mathrm{Zn}$ были вставлены два монослоя атомов $\mathrm{Cd}$ на расстоянии пяти моноатомных слоев $(\sim 15 \AA)$ друг от друга. Слои ZnTe были выращены методом молекулярнолучевой эпитаксии на подложках (001) GaAs, монослои $\mathrm{Cd}$ формировались методом атомного наслаивания. Моноатомные слои СdTe создают для носителей две туннельно связанные дельтаобразные квантовые ямы в матрице $\mathrm{ZnTe}$. 
Регистрировались спектры отражения и фотолюминесценции (ФЛ) при температурах от 5 до $100 \mathrm{~K}$ и при разных интенсивностях оптического возбуждения от 1 до $80 \mathrm{~W} / \mathrm{cm}^{2}$. Спектры ФЛ возбуждались лазерами с длиной волны $442 \mathrm{~nm}$ с максимальной интенсивностью излучения $80 \mathrm{~W} / \mathrm{cm}^{2}$ для надбарьерного возбуждения и с длиной волны $532 \mathrm{~nm}$ для подбарьерного возбуждения. Спектры регистрировались с помощью монохроматора МДР-206-2, оснащенного фотоумножителем.

На рис. 1 представлены спектр отражения и спектр ФЛ при температуре $5 \mathrm{~K}$. Особенность в спектре отражения на энергии $2.28 \mathrm{eV}$ совпадает с линиями ФЛ, что указывает на то, что эти линии связаны с собственными экситонными состояниями в двойных квантовых ямах $\mathrm{CdTe}$. Мы считаем, что линия ФЛ на энергии $2.31 \mathrm{eV}$ связана с возбужденным состоянием экситона. При слабом оптическом возбуждении на энергии $2.255 \mathrm{eV}$ наблюдается широкая полоса, связанная с донорно акцепторной рекомбинацией.

При температуре $5 \mathrm{~K}$ в спектре ФЛ на энергии $2.28 \mathrm{eV}$ наблюдался дублет линий шириной около $6 \mathrm{meV}$ каждая, расщепленных примерно на такую же величину. При предельно слабом надбарьерном возбуждении $\left(\sim 1 \mathrm{~W} / \mathrm{cm}^{2}\right)$ интегральные интенсивности коротковолновой и длинноволновой компонент дублета были одинаковы. С ростом интенсивности оптического возбуждения происходила „перекачка“ интенсивности ФЛ от коротковолновой линии в длинноволновую, и при уровне накачки $60 \mathrm{~W} / \mathrm{cm}^{2}$ интенсивность длинноволновой компоненты превышала интенсивность коротковолновой в два раза (рис. 2). При слабом подбарьерном возбуждении $\left(\sim 20 \mathrm{~W} / \mathrm{cm}^{2}\right)$ интенсивность длинноволновой компоненты дублета была всего лишь на $20 \%$

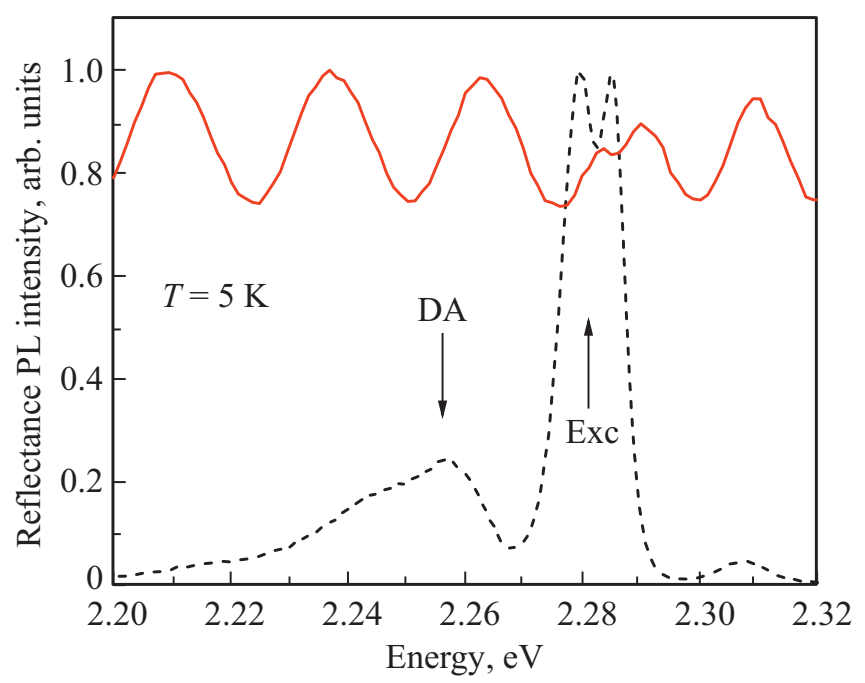

Рис. 1. Спектр фотолюминесценции исследуемой структуры (штриховая кривая), полученный при оптическом возбуждении с интенсивностью $60 \mathrm{~mW} / \mathrm{cm}^{2}$ и спектр отражения, (сплошная кривая), $T=5 \mathrm{~K}$. Стрелками отмечено положение экситонных пиков в двойной квантовой яме $\left(E_{\mathrm{xc}}\right)$ и полоса рекомбинации донорно-акцепторных пар (DA).

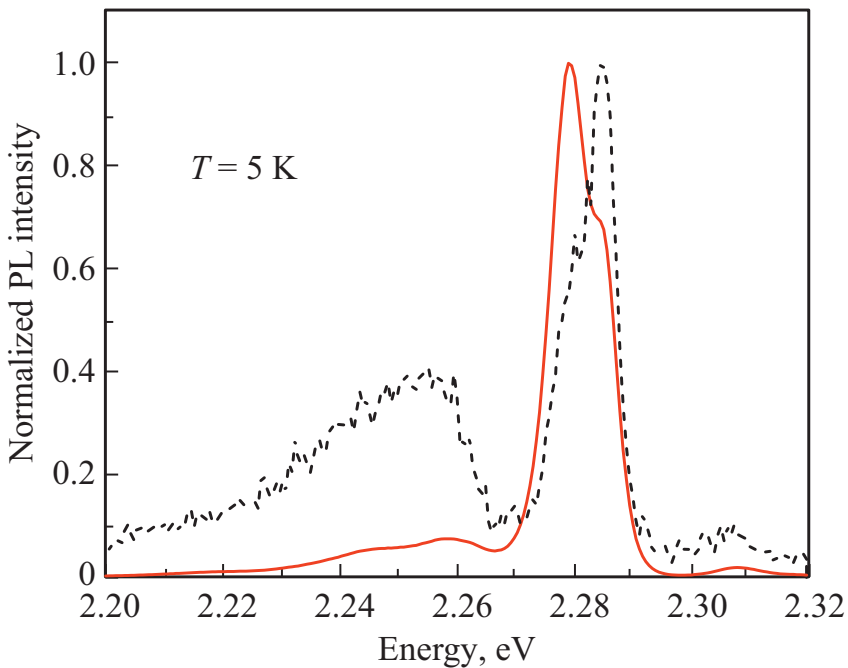

Рис. 2. Спектры фотолюминесценции при надбарьерном возбуждении $(442 \mathrm{~nm})$ с интенсивностью $80 \mathrm{~W} / \mathrm{cm}^{2}$ (сплошная кривая) и $1 \mathrm{~W} / \mathrm{cm}^{2}$ (штриховая кривая).

меньше интенсивности коротковолновой компоненты. При сильном возбуждении $\left(\sim 80 \mathrm{~W} / \mathrm{cm}^{2}\right)$ интенсивности компонент дублета выравнивались. В обоих случаях с ростом „накачки“ максимум ФЛ как бы смещался в длинноволновую сторону (рис. 2). Такое поведение выглядит необычным, как правило, с ростом „накачки“ благодаря заполнению локализованных состояний происходит перераспределение интенсивности в пользу коротковолновых компонент, то есть происходит „голубой“ сдвиг всего спектра. Различие подбарьерного и надбарьерного возбуждения связано с тем, что надбарьерное возбуждение приводит к перезарядке примесей, которая может изменить потенциал, локализующий носители, и повлиять на интенсивность линий ФЛ. При подбарьерном возбуждении этого не происходит.

Необычное поведение спектров ФЛ наблюдалось и при изменении температуры. С ростом температуры весь спектр в целом, как и ожидалось, смещался в длинноволновую сторону. Однако, коротковолновая компонента дублета смещалась быстрее длинноволновой. В результате температурные зависимости энергетического положения этих линий пересекались при температуре $65 \mathrm{~K}$. В области пересечения наблюдалось немонотонное поведение энергетического положения линий и их интенсивностей (рис. 3). Оказалось, что температурные зависимости энергий коротковолновой и длинноволновой компонент совпадают с температурными зависимостями ширины запрещенных зон ZnTe и $\mathrm{CdTe}$ соответственно [4]. Это указывает на то, что коротковолновая компонента связана со слоями ZnTe a длинноволновая со слоями CdTe.

Суммарная интенсивность этих линий падала с ростом температуры, при этом интенсивность перераспределялась от коротковолновой компоненты дублета к 


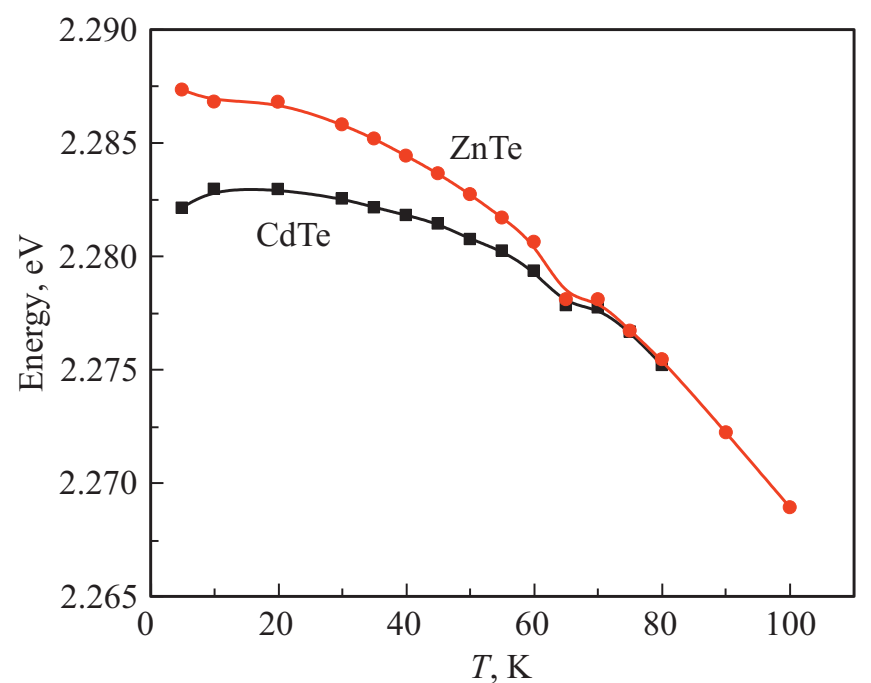

Рис. 3. Температурная зависимость энергетического положения максимумов линий тяжелого и легкого экситонов, полученная при интенсивности оптического возбуждения $\sim 60 \mathrm{~W} / \mathrm{cm}^{2}$.

длинноволновой. При этом ширина линий слабо менялась с ростом температуры.

Различие в температурных зависимостях положения этих линий указывает на то, что длинноволновая и коротковолновая компоненты дублета имеют разную природу. При этом самые простые оценки показывают, что это не могут быть симметричное и антисимметричное состояния экситона в двойной квантовой яме, так как расстояние между этими линиями слишком мало. Мы считаем, что коротковолновая компоненты связана с легким экситоном, а длинноволновая с тяжелым экситоном. (Тяжелой дыркой мы называем дырку, имеющую большую массу перпендикулярно слоям квантовой ямы, параллельно слоям она имеет малую массу.) В данной структуре расщепление легкого и тяжелого экситонов может происходить не только благодаря квантованию, но и вследствие механических напряжений. При этом, часто в структурах $\mathrm{CdTe} / \mathrm{ZnTe}$ легкий экситон из слоев CdTe вытесняется в слои ZnTe [5]. По этой причине энергии соответствующих линий и могут иметь разную температурную зависимость.

Для проверки этого были сняты спектры ФЛ от торца структуры. Правила отбора в квантовых ямах таковы [6], что оптические переходы с участием тяжелого экситона поляризованы в плоскости ямы, а переходы с участием легкого экситона имеют компоненты в обеих поляризациях.

В таком случае в спектре ФЛ, снятом из торца образца, в поляризации перпендикулярной плоскости ямы мы должны были бы видеть только линию легкого экситона. Однако, оказалось, что волноводный эффект настолько силен, что сигнал ФЛ с поляризацией вдоль слоя был на порядок больше, чем в перпендикулярной поляризации. Тем не менее, нормированный на максимум спектр показал, что в поляризации, перпендикулярной плоскости слоя, интенсивности обеих линий дублета были одинаковы. То есть, излучение тяжелого экситона выходит из образца на порядок ,лучше“, иначе в этой поляризации он не был бы виден. Это отчасти подтверждает нашу интерпретацию коротковолновой линии как линии легкого экситона, а длинноволновой линии как линии тяжелого экситона.

Однако эти качественные рассуждения не дают ответа на вопрос о точном энергетическом положении линий легкого и тяжелого экситонов и интенсивностях этих линий в спектрах ФЛ. Проблема состоит в том, что блоховские функции легкой и тяжелой дырки таковы, что для света, поляризованного вдоль слоев, вероятность перехода из подзоны легких дырок в зону проводимости в три раза меньше вероятности перехода из подзоны тяжелых дырок. В наших же спектрах интенсивности этих линий были примерно одинаковы.

\section{3. Теория}

Проведем оценку положения линий легкого и тяжелого экситона в данной структуре. Постоянные решетки ZnTe и CdTe различаются на величину $~ 7.8 \%$. В результате, помимо потенциала, связанного с так называемым „химическим“ разрывом зон, на носители действует потенциал, связанный с механическими напряжениями. Величины химического разрыва зон известны очень приблизительно. Особенно это относится к монослойным квантовым ямам, где применимость приближения эффективной массы сомнительна и, следовательно, само понятие разрыва зон является недостаточно ясно определенным. Однако, очень часто метод эффективной массы дает правильные результаты и в этом случае.

Величина полного химического разрыва зон равна разности ширин запрещенных зон ZnTe и CdTe: $\Delta E=E_{g}^{\mathrm{ZnTe}}-E_{g}^{\mathrm{CdTe}}=0.78 \mathrm{eV}$. Эта величина делится между зоной проводимости и валентной зоной. По поводу пропорции деления разрыва зон было много дискуссий и специальных измерений $[7,8]$, однако вопрос все еще остается открытым. Более того, все существующие измерения разрыва зsон проводились на достаточно толстых квантовых ямах, а эта величина может сильно отличаться для монослойных включений. Кроме того, обычно использовались не бинарные гетероструктуры, а структуры на основе твердых растворов CdZnTe. В данной работе мы, как и другие авторы [9], будем считать, что разрыв в зоне проводимости много больше разрыва в валентной зоне $\left(\Delta E_{c} \gg \Delta E_{v}\right)$, и уточним эту пропорцию, основываясь на полученных нами экспериментальных данных.

Так как буферный слой и барьерные слои имеют большую толщину, можно считать, что механические напряжения в них отрелаксировали, эти слои ненапряжены и все механические напряжения сосредоточены в 
Таблица 1. Деформационные потенциалы и упругие постоянные CdTe

\begin{tabular}{c|c|c|c|c|c}
\hline$S_{11}$ & $S_{12}$ & $S_{44}$ & $a, \mathrm{eV}$ & $b, \mathrm{eV}$ & $\sigma$ \\
\hline 3.581 & -1.394 & 5.5 & -3.85 & -1.20 & 0.9358
\end{tabular}

Таблица 2. Деформационный разрыв зон в структуре $\mathrm{CdTe} / \mathrm{ZnTe}$

\begin{tabular}{c|c|c}
\hline$\delta E_{c}$ & $\delta E_{h h}$ & $\delta E_{l h}$ \\
\hline$-150 \mathrm{meV}$ & $-20 \mathrm{meV}$ & $+160 \mathrm{meV}$
\end{tabular}

ямах. Деформационные разрывы зон можно рассчитать по формулам $[10,11$,$] :$

$$
\begin{gathered}
\delta E_{c}=2 a_{c}\left(S_{11}+2 S_{12}\right) \sigma \\
\delta E_{h h}=2 a_{v}\left(S_{11}+2 S_{12}\right) \sigma+b\left(S_{11}-S_{12}\right) \sigma \\
\delta E_{l h}=2 a_{v}\left(S_{11}+2 S_{12}\right) \sigma-b\left(S_{11}-S_{12}\right) \sigma .
\end{gathered}
$$

Здесь $\delta E_{c}$ - деформационный разрыв в зоне проводимости, $\delta E_{h h}$ - деформационный разрыв в зоне тяжелых дырок, $\delta E_{l h}$ - деформационный разрыв в зоне легких дырок, $a_{c}$ и $a_{v}$ - гидростатические деформационные потенциалы в зоне проводимости и в валентной зоне, $b$ - деформационный потенциал одноосной деформации, $S_{i j} \cdot 10^{-11} \mathrm{~m}^{2} \mathrm{~N}^{-1}$ - элементы тензора упругих постоянных, $\sigma=\frac{\varepsilon}{\left(S_{11}+S_{12}\right)}-$ механическое напряжение вдоль оси структуры, $\varepsilon=\frac{a_{j}^{L}-a_{i}^{L}}{a_{i}^{L}}-$ деформация вдоль оси структуры.

Для деформационных потенциалов справедливы соотношения [11]: $a=a_{c}-a_{v}, \frac{a_{c}}{a_{v}}=-2$. Деформационные потенциалы и упругие постоянные $\mathrm{CdTe}$ приведены в табл. 1.

Пользуясь формулой (1), можем рассчитать деформационные разрывы зон. Эти значения приведены в табл. 2 .

Деформационный разрыв зон суммируется с химическим разрывом зон, в результате получаем суммарный разрыв в зоне проводимости.

Важной характеристикой является мощность потенциала, вызванного разрывом зон. Мощность потенциала примерно равна произведению разрыва зон на постоянную решетки. Таким образом, мощность $\delta$ потенциала для электрона $\gamma=3.9 \mathrm{eV} \cdot \AA$, мощность $\delta$ потенциала для дырки $\eta=0.13 \mathrm{eV} \cdot \AA$.

\section{4. Обсуждение результатов}

В наших структурах потенциал для электрона является узким и глубоким. Однако, несмотря на то, что энергия квантования электрона в таком потенциале составляет нескольких сотен $\mathrm{meV}$, глубина проникновения волновой функции электрона в барьеры достигает $1 / d \approx 20 \AA$ из-за малой массы электрона. Это величина того же порядка, что и расстояние между ямами, то есть ямы связаны друг с другом общей волновой функцией электрона и должны рассматриваться как единое целое. Нижнее по энергии состояние является четным по отношению к отражению в плоскости, проходящей через середину расстояния между ямами. Верхнее по энергии состояние должно быть нечетным, но в нашем случае оно отсутствует (уходит выше барьеров).

Мощность $\delta$ потенциала тяжелой дырки примерно в 10 раз меньше мощности $\delta$-потенциала электрона, и следовательно, ее энергия квантования должна быть еще меньше. При этом масса дырки для движения перпендикулярно слоям в 8 раз больше массы электрона. В результате глубина проникновение волновой функции тяжелой дырки в барьеры достигает $1 / d \approx 70 \AA$.

Для легкой дырки из-за механических напряжений реализуется структура типа-II - дырка вытесняется в слои $\mathrm{ZnTe}$, заключенные между $\delta$-образными барьерами CdTe высотой порядка $150 \mathrm{meV}$. Благодаря малой толщине барьеров вероятность туннелирования дырки велика, и можно было бы считать ее почти свободной в слоях $\mathrm{ZnTe}$ если бы не было кулоновского взаимодействия с электроном.

Таким образом, главным фактором, определяющим уровни энергии и волновые функции дырок в нашей структуре, является их кулоновское взаимодействие с электроном. При этом энергия и волновые функции электрона определяются главным образом его квантованием в двойной $\delta$ образной яме, так как энергия квантования электрона во много раз превосходит его энергию взаимодействия с дыркой.

Как следует из гамильтониана Латтинжера, при квантовании в яме тяжелая дырка имеет малую массу в плоскости квантовой ямы и наоборот, легкая дырка имеет большую массу в плоскости ямы. В результате боровский радиус легкого экситона в двумерном приближении $\tilde{a}_{\mathrm{B}}$ оказывается примерно в $1.5-1.7$ раза меньше, чем боровский радиус тяжелого экситона. Следовательно, и энергия связи легкого экситона в 2D-приближении в 1.7 раза больше энергии связи тяжелого экситона. Полная энергия связи экситона в нашем приближении складывается из энергии связи двумерного экситона и энергии связи дырки в среднем поле электрона в направлении оси $z$.

Сила осциллятора 2D-экситона [12] обратно пропорциональна квадрату его боровского радиуса в плоскости и пропорциональна квадрату интеграла перекрытия волновых функций электрона и дырки вдоль оси $z$.

Оказалось, что интеграл перекрытия вдоль оси $z$ для электрона с легкой дыркой примерно равен интегралу перекрытия электрона с тяжелой дыркой. Большой интеграл перекрытия обусловлен тем, что энергия легкой дырки в $\delta$-образных барьерах выше ее энергии в промежутке меду ними. Она как бы „втягивается“ в область 


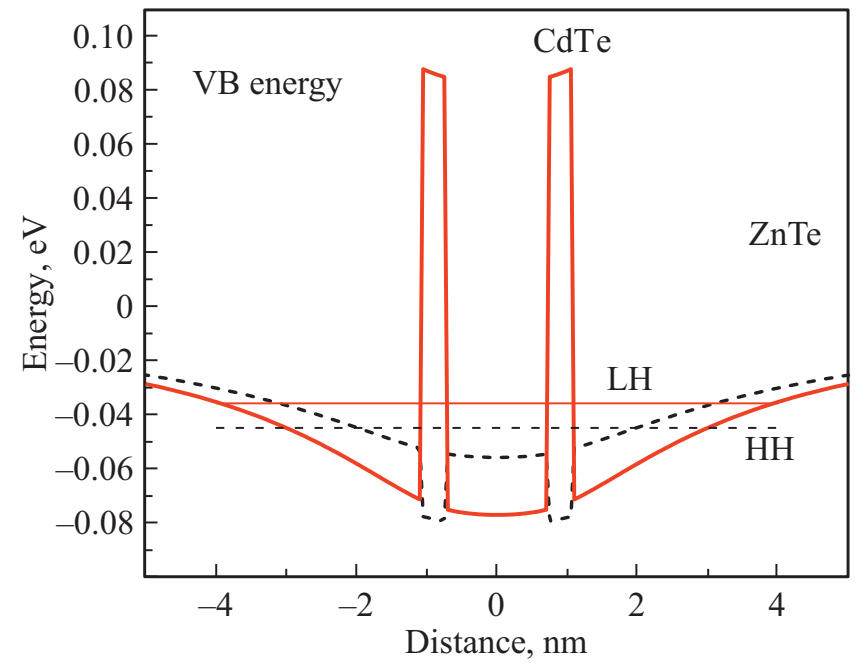

Рис. 4. Расчет эффективного потенциала вдоль оси $z$ для дырок, создаваемого электроном. Потенциал для тяжелой дырки - штриховая линия, потенциал легкой дырки - сплошная линия. Тонкими линиями НH и LH обозначены уровни энергии тяжелых и легких дырок в эффективном потенциале.

между ямами, благодаря отталкивающему характеру $\delta$-потенциала. Тяжелая дырка, наоборот, „выталкивается“ в барьеры из области кулоновского поля электрона притягивающим $\delta$-потенциалом, так как ее энергия в $\delta$-образных барьерах ниже ее энергии между барьерами (рис. 4). В результате, несмотря на то что межзонный матричный элемент дипольного момента оптического перехода для тяжелой дырки в 3 раза больше, чем для легкой, сила осциллятора легкого экситона оказалась сравнимой с силой осциллятора тяжелого экситона благодаря большему перекрытию волновых функций, как это и наблюдается в эксперименте.

Надбарьерное возбуждение в отличие от подбарьерного приводит к перезарядке примесных центров в слоях ZnTe. Поле заряженных примесей может изменить потенциал дырок в валентной зоне. Это в свою очередь, может привести к изменению перекрытия волновых функций дырок с волновой функцией электрона и, в результате, к перераспределению интенсивности линий легких и тяжелых экситонов.

Уровни энергии и волновые функции тяжелого и легкого экситона рассчитывались путем численного решения уравнения Шредингера для экситона методом сеток. Расчетная энергия легкого и тяжелого экситонов зависела от мощности потенциала в валентной зоне, а следовательно, и от величины разрыва зон. Подбирая величину разрыва зон в расчете, мы добивались наилучшего совпадения расчетных и экспериментально измеренных энергий легкого и тяжелого экситонов. В результате находилась величина полного разрыва зон в валентной зоне. Зная величину полного и деформационного разрыва зон, можно определить величину химического разрыва зон в валентной зоне в системе CdTe/ZnTe кото- рая оказалась $\sim 10 \mathrm{meV}$. Из расчета волновых функций было получено, что сила осциллятора легкого экситона в нашей структуре равна силе осциллятора тяжелого экситона с точностью до $15 \%$. Энергия связи легкого экситона оказалась даже несколько больше энергии связи тяжелого экситона.

\section{5. Заключение}

В работе исследованы спектры отражения и фотолюминесценции структуры CdTe/ZnTe c двойными $\delta$-образными квантовыми ямами. В спектрах фотолюминесценции идентифицированы линии тяжелого и легкого экситонов. Эти линии имеют разные температурные зависимости, что указывает на то, что тяжелый экситон связан со слоями CdTe, а легкий со слоями $\mathrm{ZnTe}$. Интенсивности линий фотолюминесценции тяжелого и легкого экситонов оказались примерно одинаковыми, что указывает на то, что перекрытие волновых функций легкой дырки и электрона заметно больше, чем перекрытие волновых функций тяжелой дырки и электрона. Расчет энергетических уровней и волновых функций тяжелого и легкого экситонов подтверждает предложенную интерпретацию линий. По результатам подгонки расчета к эксперименту установлена величина химического разрыва зон в валентной зоне для монослойных включений CdTe в ZnTe, которая оказалось равной 2\% от общего химического разрыва зон.

\section{Финансирование работы}

При выполнении работы ВФА, НГФ и АЮС использовалось оборудование научного парка СПбГУ в рамках проекта INI_2019, ID: 37688845. Участие в работе GK частично поддержано Национальным научным центром (гранты № 2017/25/B/ST3/02966 и 2018/30/M/ST3/00276).

\section{Конфликт интересов}

Авторы заявляют об отсутствии конфликта интересов.

\section{Список литературы}

[1] H. Mathieu, J. Allegre, A. Chatt, P. Lefebre, J.P. Faurie. Phys. Rev. B 38, 11, 7740 (1988).

[2] D.J. Dunstan, A.D. Prins, B. Gil, J.P. Faurie. Phys. Rev. B 44, 8, 4017 (1991).

[3] C. Priester, G. Allan, M. Lannoo. J. Phys. Colloques 48, C5203-C5-206 (1987).

[4] R. Passler, E. Griebl, H. Riepl, G. Lautner, S. Bauer, H. Preis, W. Gebhardt, B. Budam, D.J. As, D. Schikora, K. Lischka, K. Papagelis, S. Ves. J. Appl. Phys. 86, 8, 4403 (1999).

[5] H. Tuffigo, N. Magnea, H. Mariette, A. Wasiela, Y. Merle d'Aubigne. Phys. Rev. B 43, 8, 14629 (1991). 
[6] E.L. Ivchenko. Optical Spectroscopy of Semiconductor Nanostructures. Alpha Science Int., Harrow, UK (2005).

[7] E. Deleporte J.M. Berroir, C. Delalande, N. Magnea, H. Mariette, J. Allegre, J. Calatayud. Phys. Rev. B 45, 11, R6305 (1992).

[8] С.В. Зайцев, И.В. Седова, С.В. Сорокин, С.В. Иванов. Письма в ЖЭТФ 88, 12, 922 (2008).

[9] В.И. Козловский, В.Г. Литвинов, Ю.Г. Садофьев. ФТП 34, 8, 998 (2000).

[10] H. Mariette, F. Dal'bo, N. Magnea, G. Lentz, H. Tuffigo. Phys. Rev. B 38, 17, 12443 (1988).

[11] P. Peyla, Y. Merle d'Aubigne, A. Wasiella, R. Romestain, H. Mariette, M.D. Sturge, N. Magnea, H. Tuffigo. Phys. Rev. B 46, 3, 1557 (1992).

[12] J. Feldmann, G. Peter, E.O. Gobel, P. Dawson, K. Moore, C. Foxon, R.J. Elliott. Phys. Rev. Lett. 59, 2237 (1987).

Редактор Д.В. Жуманов 